\title{
Chance oder Risiko? Europäischer Datenschutz
}

Am 25.1.2012 legte die Europäische Kommission (EU-Kommission) den Entwurf einer Verordnung zum Schutz natürlicher Personen bei der Verarbeitung personenbezogener Daten und zum freien Datenverkehr (kurz: EU-Datenschutz-Grundverordnung bzw. EU-DS-GVO) vor. Ziele sollen die Vereinheitlichung des Datenschutzniveaus in der Europäischen Union, eine weitgehende Reduzierung des Verwaltungsaufwands für die Beteiligten und die Verbesserung der Durchsetzung von Rechten Betroffener sowie eine wirksame länderübergreifende Überwachung des Datenschutzes sein. Einige der enthaltenen Datenschutzregelungen finden sich bisher in der Richtlinie 95/46/EG zum Schutz natürlicher Personen bei der Verarbeitung personenbezogener Daten und zum freien Datenverkehr aus dem Jahr 1995 wieder. Nach Ansicht zahlreicher Kritiker wurde in den letzten Jahren mit der Richtlinie jedoch kein ausreichender Datenschutz in den Ländern erreicht bzw. umgesetzt. Im Anbetracht der vielen internationalen Vorkommnisse - als Beispiele seien nur Facebook und PRISM genannt - war es daher lediglich eine Frage der Zeit, wann zumindest auf Europaebene versucht wird, diese internationalen Datenschutzprobleme und überaus schwerwiegenden Eingriffe in die Rechte von Betroffenen in den Griff zu bekommen. Während jedoch die einen darin die Möglichkeit sehen, länderübergreifend die Schutzrechte aller Betroffenen zu stärken, sehen andere vor allem die Gefahr, dass das in Deutschland bereits vergleichsweise hohe Datenschutzniveau unterlaufen wird. Doch selbst bei aller Sympathie mit den von der EU-Datenschutz-Grundverordnung verfolgten Zielen, dürfen die zahlreichen ungeklärten Fragen nicht außer Acht gelassen werden. So weist der vorliegende Entwurf erhebliche Regelungslücken auf, eine Vielzahl an unbestimmten Rechtsbegriffen wird nicht erläutert und nur sehr selten finden sich abschließende Regelungen. Vielmehr wird zumeist der EU-Kommission die Möglichkeit von weiteren Rechtsetzungsakten geboten und dadurch Rechtsunsicherheit erheblich gefördert. Einige der derzeit in diesem Zusammenhang am häufigsten diskutierten Fragen werden daher im vorliegenden Heft aufgegriffen und beispielsweise der Kritik nachgegangen, inwieweit die Umsetzung europaweit einheitlicher Datenschutzregelungen in Form einer Verordnung Einfluss auf das nationale Recht in Deutschland hat. Darüber hinaus werden die sich aus der Verordnung ergebenden Besonderheiten im Bereich von Beschäftigtenverhältnissen und beim Einsatz von Videotechnik näher behandelt. Ebenso wird untersucht, welchen Anwendungsbereich die Verordnung im Gegensatz zum Bundesdatenschutzrecht überhaupt regelt.

Nicht alle in der EU-Datenschutz-Grundverordnung enthaltenen Rechte und Pflichten lassen sich hier näher behandeln. Die getroffene Auswahl zeigt jedoch eindringlich: Es gibt noch einen erheblichen Klärungsbedarf, dem der bisher diskutierte Entwurf der EU-Datenschutzgrundverordnung nicht gerecht wird.

Britta A. Mester 